\title{
Approximation for the Hierarchical Constrained Variational Inequalities over the Fixed Points of Nonexpansive Semigroups
}

\author{
Li-Jun Zhu \\ School of Information and Calculation, Beifang University of Nationalities, Yinchuan 750021, China \\ Correspondence should be addressed to Li-Jun Zhu; zhulijun1995@sohu.com
}

Received 22 December 2012; Accepted 6 February 2013

Academic Editor: Jen-Chih Yao

Copyright (C) 2013 Li-Jun Zhu. This is an open access article distributed under the Creative Commons Attribution License, which permits unrestricted use, distribution, and reproduction in any medium, provided the original work is properly cited.

\begin{abstract}
The purpose of the present paper is to study the hierarchical constrained variational inequalities of finding a point $x^{*}$ such that $x^{*} \in \Omega,\left\langle(A-\gamma f) x^{*}-(I-B) S x^{*}, x-x^{*}\right\rangle \geq 0, \forall x \in \Omega$, where $\Omega$ is the set of the solutions of the following variational inequality: $x^{*} \in F,\left\langle(A-S) x^{*}, x-x^{*}\right\rangle \geq 0, \forall x \in F$, where $A, B$ are two strongly positive bounded linear operators, $f$ is a $\rho$-contraction, $S$ is a nonexpansive mapping, and $F$ is the fixed points set of a nonexpansive semigroup $\{T(s)\}_{s \geq 0}$. We present a double-net convergence hierarchical to some elements in $F$ which solves the above hierarchical constrained variational inequalities.
\end{abstract}

\section{Introduction}

Let $H$ be a real Hilbert space with inner product $\langle\cdot, \cdot\rangle$ and norm $\|\cdot\|$, respectively. Let $C$ be a nonempty closed convex subset of $H$. Recall that a self-mapping $f$ of $C$ is said to be contractive if there exists a constant $\rho \in[0,1)$ such that $\|f(x)-f(y)\| \leq \rho\|x-y\|$ for all $x, y \in C$. A mapping $T: C \rightarrow C$ is called nonexpansive if

$$
\|T x-T y\| \leq\|x-y\|, \quad \forall x, y \in C .
$$

We denote by $\operatorname{Fix}(T)$ the set of fixed points of $T$; that is, $\operatorname{Fix}(T)=\{x \in C: T x=x\}$. A bounded linear operator $B$ is called strongly positive on $H$ if there exists a constant $\tilde{\gamma}>0$ such that

$$
\langle B x, x\rangle \geq \tilde{\gamma}\|x\|^{2}, \quad \forall x \in H .
$$

It is well known that the variational inequality for an operator, $\varphi: H \rightarrow H$, over a nonempty, closed, and convex set, $C \subset H$, is to find a point $x^{*} \in C$ with the property

$$
\left\langle\varphi\left(x^{*}\right), y-x^{*}\right\rangle \geq 0, \quad \forall y \in C .
$$

The set of the solutions of the variational inequality (3) is denoted by $\operatorname{VI}(C, \varphi)$. If the mapping $\varphi$ is a monotone operator, then we say that $\mathrm{VI}(3)$ is monotone. It is well known that if $\varphi$ is Lipschitzian and strongly monotone, then for small enough $\delta>0$, the mapping $P_{C}(I-\delta \varphi)$ is a contraction on $C$ and so the sequence $\left\{x_{n}\right\}$ of Picard iterates, given by $x_{n}=P_{C}(I-\delta \varphi) x_{n-1}(n \geq 1)$, converges strongly to the unique solution of the $\mathrm{VI}(3)$. This sort of $\mathrm{VI}(3)$ where $\varphi$ is strongly monotone and Lipschitzian is originated from Yamada [1]. However, if $\varphi$ is only monotone (not strongly monotone), then their iterative methods do not apply to VI.

Many practical problems such as signal processing and network resource allocation are formulated as the variational inequality over the set of the solutions of some nonlinear mappings (e.g., the fixed point set of nonexpansive mappings), and algorithms to solve these problems have been proposed. Iterative algorithms have been presented for the convex optimization problem with a fixed point constraint along with proof that these algorithms strongly converge to the unique solution of problems with a strongly monotone operator. The strong monotonicity condition guarantees the uniqueness of the solution. For some related works on the variational inequalities, please see [2-23] and the references therein. Particulary, the variational inequality problems over the fixed points of nonexpansive mappings have been considered. The reader can consult $[16,24]$. On the other hand, we note that in the literature, nonlinear ergodic theorems for nonexpansive semigroups have been considered by many authors; see, for example, [25-32]. In this paper, we will 
consider a general variational inequality problem with the variational inequality constraint is the fixed points of nonexpansive semigroups.

The purpose of the present paper is to study the hierarchical constrained variational inequalities of finding a point $x^{*}$ such that

$$
\begin{array}{r}
x^{*} \in \Omega, \quad\left\langle(A-\gamma f) x^{*}-(I-B) S x^{*}, x-x^{*}\right\rangle \geq 0, \\
\forall x \in \Omega,
\end{array}
$$

where $\Omega$ is the set of the solutions of the following variational inequality:

$$
x^{*} \in F, \quad\left\langle(A-S) x^{*}, x-x^{*}\right\rangle \geq 0, \quad \forall x \in F,
$$

where $A$ and $B$ are two strongly positive bounded linear operators, $f$ is a $\rho$-contraction, $S$ is a nonexpansive mapping, and $F$ is the fixed points set of a nonexpansive semigroup $\{T(s)\}_{s \geq 0}$. We present a double-net convergence hierarchical to some elements in $F$ which solves the above hierarchical constrained variational inequalities.

\section{Preliminaries}

Let $C$ be a nonempty closed convex subset of a real Hilbert space $H$. The metric (or the nearest point) projection from $H$ onto $C$ is the mapping $P_{C}: H \rightarrow C$ which assigns to each point $x \in C$ the unique point $P_{C} x \in C$ satisfying the property

$$
\left\|x-P_{C} x\right\|=\inf _{y \in C}\|x-y\|=: d(x, C) .
$$

It is well known that $P_{C}$ is a nonexpansive mapping and satisfies

$$
\left\langle x-y, P_{C} x-P_{C} y\right\rangle \geq\left\|P_{C} x-P_{C} y\right\|^{2}, \quad \forall x, y \in H .
$$

Moreover, $P_{C}$ is characterized by the following property:

$$
\left\langle x-P_{C} x, y-P_{C} x\right\rangle \leq 0, \quad \forall x \in H, y \in C .
$$

Recall that a family $\{T(s)\}_{s \geq 0}$ of mappings of $H$ into itself is called a nonexpansive semigroup if it satisfies the following conditions:

(S1) $T(0) x=x$ for all $x \in H$;

(S2) $T(s+t)=T(s) T(t)$ for all $s, t \geq 0$;

(S3) $\|T(s) x-T(s) y\| \leq\|x-y\|$ for all $x, y \in H$ and $s \geq 0$;

(S4) for all $x \in H, s \rightarrow T(s) x$ is continuous.

We denote by Fix $(T(s))$ the set of fixed points of $T(s)$ and by $F$ the set of all common fixed points of $\{T(s)\}_{s \geq 0}$; that is, $F=\bigcap_{s \geq 0} \operatorname{Fix}(T(s))$. It is known that $F$ is closed and convex.

We need the following lemmas for proving our main results.

Lemma 1 (see [33]). Let $C$ be a nonempty bounded closed convex subset of a Hilbert space $H$ and let $\{T(s)\}_{s \geq 0}$ be a nonexpansive semigroup on $C$. Then, for every $h \geq 0$,

$$
\lim _{t \rightarrow \infty} \sup _{x \in C}\left\|\frac{1}{t} \int_{0}^{t} T(s) x d s-T(h) \frac{1}{t} \int_{0}^{t} T(s) x d s\right\|=0 .
$$

Lemma 2 (see [34]). Let $C$ be a closed convex subset of a real Hilbert space $H$ and let $S: C \rightarrow C$ be a nonexpansive mapping. Then, the mapping $I-S$ is demiclosed. That is, if $\left\{x_{n}\right\}$ is a sequence in $C$ such that $x_{n} \rightarrow x^{*}$ weakly and $(I-S) x_{n} \rightarrow$ $y$ strongly, then $(I-S) x^{*}=y$.

Lemma 3 (see [35]). Let $C$ be a nonempty closed convex subset of a real Hilbert space $H$. Assume that a mapping $F: C \rightarrow H$ is monotone and weakly continuous along segments (i.e., $F(x+$ ty) $\rightarrow F(x)$ weakly, as $t \rightarrow 0$, whenever $x+t y \in C$ for $x, y \in C)$. Then the variational inequality

$$
x^{*} \in C, \quad\left\langle F x^{*}, x-x^{*}\right\rangle \geq 0, \quad \forall x \in C,
$$

is equivalent to the dual variational inequality

$$
x^{*} \in C, \quad\left\langle F x, x-x^{*}\right\rangle \geq 0, \quad \forall x \in C .
$$

\section{Main Results}

Now we consider the following hierarchical variational inequality with the variational inequality constraint over the fixed points set of nonexpansive semigroups $\{T(s)\}_{s \geq 0}$.

Problem 1. Let $C$ be a nonempty closed convex subset of a real Hilbert space $H$. Let $f: C \rightarrow H$ be a $\rho$-contraction with coefficient $\rho \in[0,1)$ and let $S: C \rightarrow C$ be a nonexpansive mapping. Let $\{T(s)\}_{s>0}$ be a nonexpansive semigroup on $C$ and let $A, B: H \rightarrow H$ be two strongly positive bounded linear operators with coefficients $\tilde{\lambda}(1 \leq \tilde{\lambda}<2)$ and $\tilde{\gamma}(0<$ $\tilde{\gamma}<1$ ), respectively. Let $\gamma$ be a constant satisfying $0<\gamma \rho<\tilde{\gamma}$.

Now, our objective is to find $x^{*}$ such that

$$
x^{*} \in \Omega, \quad\left\langle(A-\gamma f) x^{*}-(I-B) S x^{*}, x-x^{*}\right\rangle \geq 0,
$$$$
\forall x \in \Omega \text {, }
$$

where $\Omega:=\operatorname{VI}(F, A-S)$ is the set of the solutions of the following variational inequality:

$$
x^{*} \in F, \quad\left\langle(A-S) x^{*}, x-x^{*}\right\rangle \geq 0, \quad \forall x \in F .
$$

We observe that $(A-\gamma f)-(I-B) S$ is strongly monotone and Lipschitz continuous. In fact, we have

$$
\begin{aligned}
&\langle(A-\gamma f) x-(I-B) S x-[(A-\gamma f) y-(I-B) S y], x-y\rangle \\
&=\langle A x-A y, x-y\rangle-\gamma\langle f(x)-f(y), x-y\rangle \\
&-(I-B)\langle S x-S y, x-y\rangle \geq \tilde{\lambda}\|x-y\|^{2}-\gamma \rho\|x-y\|^{2} \\
&-(1-\tilde{\gamma})\|x-y\|^{2}=(\tilde{\lambda}-1+\tilde{\gamma}-\gamma \rho)\|x-y\|^{2}, \\
& \|(A-\gamma f) x-(I-B) S x-[(A-\gamma f) y-(I-B) S y] \| \\
& \leq\|A x-A y\|+\gamma\|f(x)-f(y)\|+\|I-B\|\|S x-S y\| \\
& \leq\|A\|\|x-y\|+\gamma \rho\|x-y\|+(1-\tilde{\gamma})\|x-y\| \\
&=(1+\|A\|+\gamma \rho-\tilde{\gamma})\|x-y\| .
\end{aligned}
$$


Hence, the existence and the uniqueness of the solution to Problem 1 are guaranteed.

In order to solve the above hierarchical constrainted variational inequality, we present the following double net.

Algorithm 4. Set $\kappa=1 /(\widetilde{\lambda}+\widetilde{\gamma}-\gamma \rho)$. Then, $0<\kappa \leq 1$. For each $(s, t) \in(0, \kappa) \times(0,1)$, we define a double net $\left\{x_{s, t}\right\}$ implicitly by

$$
\begin{aligned}
x_{s, t}=P_{C}[ & s\left(t \gamma f\left(x_{s, t}\right)+(I-t B) S x_{s, t}\right) \\
& \left.+(I-s A) \frac{1}{\lambda_{s}} \int_{0}^{\lambda_{s}} T(\nu) x_{s, t} d \nu\right] .
\end{aligned}
$$

Note that this implicit manner algorithm is well defined. In fact, we define the mapping

$$
\begin{array}{r}
x \longmapsto W_{s, t}(x):=P_{C}[s(t \gamma f(x)+(I-t B) S x) \\
\left.+(I-s A) \frac{1}{\lambda_{s}} \int_{0}^{\lambda_{s}} T(\nu) x d \nu\right], \\
(s, t) \in(0, \kappa) \times(0,1) .
\end{array}
$$

Note that this self-mapping is a contraction. As a matter of fact, we have

$$
\begin{aligned}
& \left\|W_{s, t}(x)-W_{s, t}(y)\right\| \\
& =\| P_{C}[s(t \gamma f(x)+(I-t B) S x) \\
& \left.+(I-s A) \frac{1}{\lambda_{s}} \int_{0}^{\lambda_{s}} T(\nu) x d \nu\right] \\
& -P_{C}[s(t \gamma f(y)+(I-t B) S y) \\
& \left.+(I-s A) \frac{1}{\lambda_{s}} \int_{0}^{\lambda_{s}} T(\nu) y d \nu\right] \| \\
& \leq s t \gamma\|f(x)-f(y)\| \\
& +s\|I-t B\|\|S x-S y\| \\
& +\|I-s A\| \| \frac{1}{\lambda_{s}} \int_{0}^{\lambda_{s}} T(\nu) x d \nu \\
& -\frac{1}{\lambda_{s}} \int_{0}^{\lambda_{s}} T(\nu) y d \nu \| \\
& \leq[1-(\tilde{\lambda}-1) s-(\tilde{\gamma}-\gamma \rho) s t]\|x-y\| .
\end{aligned}
$$

Since $(s, t) \in(0, \kappa) \times(0,1), 0<1-(\tilde{\lambda}-1) s-(\widetilde{\gamma}-$ $\gamma \rho) s t<1$. Hence, $W_{s, t}$ is a contraction. Therefore, by Banach's Contraction Principle, $W_{s, t}$ has a unique fixed point which is denoted by $x_{s, t} \in C$.

Next we show the behavior of the net $\left\{x_{s, t}\right\}$ as $s \rightarrow 0$ and $t \rightarrow 0$ successively.
Theorem 5. Assume that $V I(F, A-S) \neq \emptyset$. Then, for each fixed $t \in(0,1)$, the net $\left\{x_{s, t}\right\}$ defined by (15) converges in norm, as $s \rightarrow 0+$, to a solution $x_{t} \in F$. Moreover, as $t \rightarrow 0+$, the net $\left\{x_{t}\right\}$ converges in norm to the unique solution $x^{*}$ of Problem 1 .

Proof. We first show that the sequence $\left\{x_{s, t}\right\}$ is bounded. Take $y^{*} \in F$. From (15), we have

$$
\begin{aligned}
& \left\|x_{s, t}-y^{*}\right\|=\| P_{c}\left[s\left(t \gamma f\left(x_{s, t}\right)+(I-t B) S x_{s, t}\right)\right. \\
& \left.+(I-s \mathrm{~A}) \frac{1}{\lambda_{s}} \int_{0}^{\lambda_{s}} T(\nu) x d \nu\right]-y^{*} \| \\
& \leq \| s\left(t \gamma f\left(x_{s, t}\right)+(I-t B) S x_{s, t}\right) \\
& +(I-s A) \frac{1}{\lambda_{s}} \int_{0}^{\lambda_{s}} T(\nu) x d v-y^{*} \\
& =\| \operatorname{st} \gamma\left(f\left(x_{s, t}\right)-f\left(y^{*}\right)\right) \\
& +s(I-t B)\left(S x_{s, t}-S y^{*}\right) \\
& +\operatorname{st} \gamma f\left(y^{*}\right)+s(I-t B) S y^{*}-s A y^{*} \\
& +(I-s A)\left(\frac{1}{\lambda_{s}} \int_{0}^{\lambda_{s}} T(\nu) x d \nu-y^{*}\right) \| \\
& \leq \operatorname{st} \gamma\left\|f\left(x_{s, t}\right)-f\left(y^{*}\right)\right\| \\
& +s\|I-t B\|\left\|S x_{s, t}-S y^{*}\right\| \\
& +\|I-s A\| \times\left\|\frac{1}{\lambda_{s}} \int_{0}^{\lambda_{s}} T(\nu) x d \nu-y^{*}\right\| \\
& +s\left\|t \gamma f\left(y^{*}\right)+(I-t B) S y^{*}-A y^{*}\right\| \\
& \leq \operatorname{st} \gamma \rho\left\|x_{s, t}-y^{*}\right\|+s(1-t \widetilde{\gamma})\left\|x_{s, t}-y^{*}\right\| \\
& +(1-s \widetilde{\lambda})\left\|x_{s, t}-y^{*}\right\| \\
& +s\left\|t \gamma f\left(y^{*}\right)+(I-t B) S y^{*}-A y^{*}\right\| \\
& =[1-(\tilde{\lambda}-1) s-(\tilde{\gamma}-\gamma \rho) s t]\left\|x_{s, t}-y^{*}\right\| \\
& +s\left\|t \gamma f\left(y^{*}\right)+(I-t B) S y^{*}-A y^{*}\right\| \text {. }
\end{aligned}
$$

Hence

$$
\begin{aligned}
\left\|x_{s, t}-y^{*}\right\| \leq & \frac{1}{(\widetilde{\gamma}-\gamma \rho) t+\tilde{\lambda}-1} \\
& \times\left\|t \gamma f\left(y^{*}\right)+(I-t B) S y^{*}-A y^{*}\right\| .
\end{aligned}
$$

It follows that for each fixed $t \in(0,1),\left\{x_{s, t}\right\}$ is bounded.

Next, we show that $\lim _{s \rightarrow 0}\left\|T(\tau) x_{s, t}-x_{s, t}\right\|=0$ for all $0 \leq \tau<\infty$ and consequently, as $s \rightarrow 0+$, the entire net $\left\{x_{s, t}\right\}$ converges in norm to $x_{t} \in F$. 
For each fixed $t \in(0,1)$, we set $R_{t}:=(1 /((\tilde{\gamma}-\gamma \rho) t+\tilde{\lambda}-$ 1)) $\left\|t \gamma f\left(y^{*}\right)+(I-t B) S y^{*}-A y^{*}\right\|$. It is clear that for each fixed $t \in(0,1),\left\{x_{s, t}\right\} \subset B\left(y^{*}, R_{t}\right)$. Notice that

$$
\left\|\frac{1}{\lambda_{s}} \int_{0}^{\lambda_{s}} T(v) x_{s, t} d v-y^{*}\right\| \leq\left\|x_{s, t}-y^{*}\right\| \leq R_{t}
$$

Moreover, we observe that if $x \in B\left(y^{*}, R_{t}\right)$, then

$$
\left\|T(s) x-y^{*}\right\| \leq\left\|T(s) x-T(s) y^{*}\right\| \leq\left\|x-y^{*}\right\| \leq R_{t},
$$

that is, $B\left(y^{*}, R_{t}\right)$ is $T(s)$-invariant for all $s$.

From (15), we deduce

$$
\begin{aligned}
& \left\|T(\tau) x_{s, t}-x_{s, t}\right\| \\
& \leq\left\|T(\tau) x_{s, t}-T(\tau) \frac{1}{\lambda_{s}} \int_{0}^{\lambda_{s}} T(\nu) x_{s, t} d \nu\right\| \\
& +\left\|T(\tau) \frac{1}{\lambda_{s}} \int_{0}^{\lambda_{s}} T(\nu) x_{s, t} d \nu-\frac{1}{\lambda_{s}} \int_{0}^{\lambda_{s}} T(\nu) x_{s, t} d \nu\right\| \\
& +\left\|\frac{1}{\lambda_{s}} \int_{0}^{\lambda_{s}} T(v) x_{s, t} d v-x_{s, t}\right\| \\
& \leq\left\|T(\tau) \frac{1}{\lambda_{s}} \int_{0}^{\lambda_{s}} T(\nu) x_{s, t} d v-\frac{1}{\lambda_{s}} \int_{0}^{\lambda_{s}} T(\nu) x_{s, t} d v\right\| \\
& +2\left\|x_{s, t}-\frac{1}{\lambda_{s}} \int_{0}^{\lambda_{s}} T(\nu) x_{s, t} d \nu\right\| \\
& \leq 2 s \| t \gamma f\left(x_{s, t}\right)+(I-t B) S x_{s, t} \\
& -\frac{A}{\lambda_{s}} \int_{0}^{\lambda_{s}} T(\nu) x_{s, t} d \nu \| \\
& +\left\|T(\tau) \frac{1}{\lambda_{s}} \int_{0}^{\lambda_{s}} T(\nu) x_{s, t} d \nu-\frac{1}{\lambda_{s}} \int_{0}^{\lambda_{s}} T(\nu) x_{s, t} d \nu\right\| .
\end{aligned}
$$

Since $\left\{x_{s, t}\right\}$ is bounded, $\left\{f\left(x_{s, t}\right)\right\}$ and $\left\{S x_{s, t}\right\}$ are also bounded. Then, from Lemma 1 , we deduce for all $0 \leq \tau<\infty$ and fixed $t \in(0,1)$ that

$$
\lim _{s \rightarrow 0}\left\|T(\tau) x_{s, t}-x_{s, t}\right\|=0
$$

Set $y_{s, t}=s\left(t \gamma f\left(x_{s, t}\right)+(I-t B) S x_{s, t}\right)+(I-s A)\left(1 / \lambda_{s}\right)$ $\int_{0}^{\lambda_{s}} T(\nu) x_{s, t} d v$ for all $(s, t) \in(0, \kappa) \times(0,1)$. We then have $x_{s, t}=P_{C} y_{s, t}$, and for any $y^{*} \in F$,

$$
\begin{aligned}
x_{s, t}-y^{*}= & x_{s, t}-y_{s, t}+y_{s, t}-y^{*} \\
= & x_{s, t}-y_{s, t}+s\left(t \gamma f\left(x_{s, t}\right)+(I-t B) S x_{s, t}\right) \\
& +(I-s A) \frac{1}{\lambda_{s}} \int_{0}^{\lambda_{s}} T(\nu) x_{s, t} d v-y^{*}=x_{s, t}-y_{s, t} \\
& +s t \gamma\left(f\left(x_{s, t}\right)-f\left(y^{*}\right)\right)+s(I-t B)\left(S x_{s, t}-S y^{*}\right) \\
& +s\left(t \gamma f\left(y^{*}\right)+(I-t B) S y^{*}-A y^{*}\right) \\
& +(I-s A)\left(\frac{1}{\lambda_{s}} \int_{0}^{\lambda_{s}} T(\nu) x_{s, t} d v-y^{*}\right) .
\end{aligned}
$$

Notice that

$$
\left\langle x_{s, t}-y_{s, t}, x_{s, t}-y^{*}\right\rangle \leq 0 .
$$

Thus, we have

$$
\begin{aligned}
\left\|x_{s, t}-y^{*}\right\|^{2}= & \left\langle x_{s, t}-y_{s, t}, x_{s, t}-y^{*}\right\rangle \\
& +s t \gamma\left\langle f\left(x_{s, t}\right)-f\left(y^{*}\right), x_{s, t}-y^{*}\right\rangle \\
& +s(I-t B)\left\langle S x_{s, t}-S y^{*}, x_{s, t}-y^{*}\right\rangle \\
& +(I-s A) \\
& \times\left\langle\frac{1}{\lambda_{s}} \int_{0}^{\lambda_{s}} T(v) x_{s, t} d v-y^{*}, x_{s, t}-y^{*}\right\rangle \\
& +s\left\langle t \gamma f\left(y^{*}\right)+(I-t B) S y^{*}-A y^{*}, x_{s, t}-y^{*}\right\rangle \\
\leq & s t \gamma\left\|f\left(x_{s, t}\right)-f\left(y^{*}\right)\right\|\left\|x_{s, t}-y^{*}\right\| \\
& +s\|I-t B\|\left\|S x_{s, t}-S y^{*}\right\|\left\|x_{s, t}-y^{*}\right\| \\
& +s\left\langle t \gamma f\left(y^{*}\right)+(I-t B) S y^{*}-A y^{*}, x_{s, t}-y^{*}\right\rangle \\
& +\|I-s A\|\left\|\frac{1}{\lambda_{s}} \int_{0}^{\lambda_{s}} T(\nu) x_{s, t} d \nu-y^{*}\right\|\left\|x_{s, t}-y^{*}\right\| \\
\leq & s t \gamma \rho\left\|x_{s, t}-y^{*}\right\|^{2}+s(1-t \widetilde{\gamma})\left\|x_{s, t}-y^{*}\right\|^{2} \\
& +s\left\langle t \gamma f\left(y^{*}\right)+(I-t B) S y^{*}-A y^{*}, x_{s, t}-y^{*}\right\rangle \\
& +(1-s \widetilde{\lambda})\left\|x_{s, t}-y^{*}\right\|^{2} \\
= & {[1-(\widetilde{\lambda}-1) s-(\widetilde{\gamma}-\gamma \rho) s t]\left\|x_{s, t}-y^{*}\right\|^{2} } \\
& +s\left\langle t \gamma f\left(y^{*}\right)+(I-t B) S y^{*}-A y^{*}, x_{s, t}-y^{*}\right\rangle .
\end{aligned}
$$


So

$$
\begin{aligned}
&\left\|x_{s, t}-y^{*}\right\|^{2} \leq \frac{1}{(\widetilde{\gamma}-\gamma \rho) t+\widetilde{\lambda}-1} \\
& \times\left\langle t \gamma f\left(y^{*}\right)+(I-t B) S y^{*}-A y^{*}, x_{s, t}-y^{*}\right\rangle, \\
& y^{*} \in F .
\end{aligned}
$$

Assume $\left\{s_{n}\right\} \subset(0,1)$ such that $s_{n} \rightarrow 0$ as $n \rightarrow \infty$. By (27), we obtain immediately that

$$
\begin{aligned}
&\left\|x_{s_{n}, t}-y^{*}\right\|^{2} \leq \frac{1}{(\tilde{\gamma}-\gamma \rho) t+\tilde{\lambda}-1} \\
& \times\left\langle t \gamma f\left(y^{*}\right)+(I-t B) S y^{*}-A y^{*}, x_{s_{n}, t}-y^{*}\right\rangle, \\
& y^{*} \in F .
\end{aligned}
$$

Since $\left\{x_{s_{n}, t}\right\}$ is bounded, there exists a subsequence $\left\{s_{n_{i}}\right\}$ of $\left\{s_{n}\right\}$ such that $\left\{x_{s_{n_{i}}, t}\right\}$ converges weakly to a point $x_{t}$. From (23) and Lemma 2, we get $x_{t} \in F$. We can substitute $x_{t}$ for $y^{*}$ in (28) to get

$$
\begin{aligned}
\left\|x_{s_{n}, t}-x_{t}\right\|^{2} \leq & \frac{1}{(\tilde{\gamma}-\gamma \rho) t+\tilde{\lambda}-1} \\
& \times\left\langle t \gamma f\left(x_{t}\right)+(I-t B) S x_{t}-A x_{t}, x_{s_{n}, t}-x_{t}\right\rangle .
\end{aligned}
$$

The weak convergence of $\left\{x_{s_{n}, t}\right\}$ to $x_{t}$ actually implies that $x_{s_{n}, t} \rightarrow x_{t}$ strongly. This has proved the relative normcompactness of the net $\left\{x_{s, t}\right\}$ as $s \rightarrow 0+$ for each fixed $t \in(0,1)$.

In (28), we take the limit as $n \rightarrow \infty$ to get

$$
\begin{aligned}
\left\|x_{t}-y^{*}\right\|^{2} \leq & \frac{1}{(\tilde{\gamma}-\gamma \rho) t+\tilde{\lambda}-1} \\
& \times\left\langle t \gamma f\left(y^{*}\right)+(I-t B) S y^{*}-A y^{*}, x_{t}-y^{*}\right\rangle, \\
& \forall y^{*} \in F .
\end{aligned}
$$

In particular, $x_{t}$ solves the following variational inequality:

$$
\begin{aligned}
x_{t} \in F, \quad\left\langle A y^{*}-t \gamma f\left(y^{*}\right)\right. & \\
& \left.-(I-t B) S y^{*}, y^{*}-x_{t}\right\rangle \geq 0, \quad \forall y^{*} \in F .
\end{aligned}
$$

Note that the mapping $A-t \gamma f-(I-t B) S$ is monotone for all $t \in(0,1)$, since

$$
\begin{aligned}
&\langle A x-t \gamma f(x)-(I-t B) S x \\
&-(A y-t \gamma f(y)-(I-t B) S y), x-y\rangle \\
&=\langle A x-A y, x-y\rangle-t \gamma\langle f(x)-f(y), x-y\rangle \\
&-(I-t B)\langle S x-S y, x-y\rangle \geq \tilde{\lambda}\|x-y\|^{2} \\
&-t \gamma \rho\|x-y\|^{2}-(1-t \tilde{\gamma})\|x-y\|^{2} \\
&= {[\widetilde{\lambda}-1+(\widetilde{\gamma}-\gamma \rho) t]\|x-y\|^{2} \geq 0 . }
\end{aligned}
$$

By Lemma 3, (31) is equivalent to its dual VI:

$$
\begin{array}{r}
x_{t} \in F, \quad\left\langle A x_{t}-t \gamma f\left(x_{t}\right)-(I-t B) S x_{t}, y^{*}-x_{t}\right\rangle \geq 0, \\
\forall y^{*} \in F .
\end{array}
$$

Next we show that as $s \rightarrow 0+$, the entire net $\left\{x_{s, t}\right\}$ converges in norm to $x_{t} \in F$. We assume $x_{s_{n}^{\prime}, t} \rightarrow x_{t}^{\prime}$, where $s_{n}^{\prime} \rightarrow 0$. Similarly, by the above proof, we deduce $x_{t}^{\prime} \in F$ which solves the following variational inequality:

$$
\begin{array}{r}
x_{t}^{\prime} \in F, \quad\left\langle A x_{t}^{\prime}-t \gamma f\left(x_{t}^{\prime}\right)-(I-t B) S x_{t}^{\prime}, y^{*}-x_{t}^{\prime}\right\rangle \geq 0, \\
\forall y^{*} \in F .
\end{array}
$$

In (33), we take $y^{*}=x_{t}^{\prime}$ to get

$$
\left\langle A x_{t}-t \gamma f\left(x_{t}\right)-(I-t B) S x_{t}, x_{t}^{\prime}-x_{t}\right\rangle \geq 0 .
$$

In (34), we take $y^{*}=x_{t}$ to get

$$
\left\langle A x_{t}^{\prime}-t \gamma f\left(x_{t}^{\prime}\right)-(I-t B) S x_{t}^{\prime}, x_{t}-x_{t}^{\prime}\right\rangle \geq 0 .
$$

Adding up (35) and (36) yields

$$
\begin{aligned}
& \left\langle A x_{t}-A x_{t}^{\prime}, x_{t}-x_{t}^{\prime}\right\rangle-t \gamma\left\langle f\left(x_{t}\right)-f\left(x_{t}^{\prime}\right), x_{t}-x_{t}^{\prime}\right\rangle \\
& -(I-t B)\left\langle S x_{t}-S x_{t}^{\prime}, x_{t}-x_{t}^{\prime}\right\rangle \leq 0 .
\end{aligned}
$$

At the same time we note that

$$
\begin{aligned}
\left\langle A x_{t}-A x_{t}^{\prime}, x_{t}-x_{t}^{\prime}\right\rangle-t \gamma\left\langle f\left(x_{t}\right)-f\left(x_{t}^{\prime}\right), x_{t}-x_{t}^{\prime}\right\rangle \\
\quad-(I-t B)\left\langle S x_{t}-S x_{t}^{\prime}, x_{t}-x_{t}^{\prime}\right\rangle \\
\geq[\tilde{\lambda}-1+(\tilde{\gamma}-\gamma \rho) t]\left\|x_{t}-x_{t}^{\prime}\right\|^{2} \\
\geq 0 .
\end{aligned}
$$

Therefore, by (37) and (38), we deduce

$$
x_{t}^{\prime}=x_{t} .
$$


Hence the entire net $\left\{x_{s, t}\right\}$ converges in norm to $x_{t} \in F$ as $s \rightarrow 0+$.

As $t \rightarrow 0+$, the net $\left\{x_{t}\right\}$ converges to the unique solution $x^{*}$ of Problem 1 .

In (33), we take any $y^{*} \in \Omega$ to deduce

$$
\left\langle A x_{t}-t \gamma f\left(x_{t}\right)-(I-t B) S x_{t}, y^{*}-x_{t}\right\rangle \geq 0 .
$$

By virtue of the monotonicity of $A-S$ and the fact that $y^{*} \in \Omega$, we have

$$
\left\langle A x_{t}-S x_{t}, y^{*}-x_{t}\right\rangle \leq\left\langle A y^{*}-S y^{*}, y^{*}-x_{t}\right\rangle \leq 0 .
$$

We can rewrite (40) as

$$
\begin{aligned}
& \left\langle t\left[A x_{t}-\gamma f\left(x_{t}\right)-(I-B) S x_{t}\right]\right. \\
& \left.\quad+(1-t)\left(A x_{t}-S x_{t}\right), y^{*}-x_{t}\right\rangle \geq 0 .
\end{aligned}
$$

It follows from (41) and (42) that

$$
\left\langle A x_{t}-\gamma f\left(x_{t}\right)-(I-B) S x_{t}, y^{*}-x_{t}\right\rangle \geq 0, \quad \forall y^{*} \in \Omega .
$$

Hence

$$
\begin{aligned}
\left\|x_{t}-y^{*}\right\|^{2} \leq & \left\langle x_{t}-y^{*}, x_{t}-y^{*}\right\rangle \\
& +\left\langle\gamma f\left(x_{t}\right)+(I-B) S x_{t}-A x_{t}, x_{t}-y^{*}\right\rangle \\
= & \gamma\left\langle f\left(x_{t}\right)-f\left(y^{*}\right), x_{t}-y^{*}\right\rangle \\
& +(I-B)\left\langle S x_{t}-S y^{*}, x_{t}-y^{*}\right\rangle \\
& +\left\langle A y^{*}-A x_{t}, x_{t}-y^{*}\right\rangle \\
& +\left\langle\gamma f\left(y^{*}\right)+(I-B) S y^{*}-A y^{*}, x_{t}-y^{*}\right\rangle \\
\leq & \gamma \rho\left\|x_{t}-y^{*}\right\|^{2}+(1-\widetilde{\gamma})\left\|x_{t}-y^{*}\right\|^{2} \\
& -\tilde{\lambda}\left\|x_{t}-y^{*}\right\|^{2} \\
& +\left\langle\gamma f\left(y^{*}\right)+(I-B) S y^{*}-A y^{*}, x_{t}-y^{*}\right\rangle \\
= & {[1-(\tilde{\lambda}+\tilde{\gamma}-\gamma \rho)]\left\|x_{t}-y^{*}\right\|^{2} } \\
& +\left\langle\gamma f\left(y^{*}\right)+(I-B) S y^{*}-A y^{*}, x_{t}-y^{*}\right\rangle .
\end{aligned}
$$

Therefore,

$$
\begin{aligned}
&\left\|x_{t}-y^{*}\right\|^{2} \leq \frac{1}{\tilde{\lambda}+\tilde{\gamma}-\gamma \rho} \\
& \times\left\langle\gamma f\left(y^{*}\right)+(I-B) S y^{*}-A y^{*}, x_{t}-y^{*}\right\rangle, \\
& y^{*} \in \Omega .
\end{aligned}
$$

In particular,

$$
\begin{aligned}
&\left\|x_{t}-y^{*}\right\| \leq \frac{1}{\tilde{\lambda}+\tilde{\gamma}-\gamma \rho} \\
& \times\left\|\gamma f\left(y^{*}\right)+(I-B) S y^{*}-A y^{*}\right\|, \\
& \forall t \in(0,1),
\end{aligned}
$$

which implies that $\left\{x_{t}\right\}$ is bounded.
We next prove that $\omega_{w}\left(x_{t}\right) \subset \Omega$; namely, if $\left(t_{n}\right)$ is a null sequence in $(0,1)$ such that $x_{t_{n}} \rightarrow x^{\prime}$ weakly as $n \rightarrow \infty$, then $x^{\prime} \in \Omega$. To see this, we use (33) to get

$$
\begin{aligned}
& \left\langle(A-S) x_{t}, y^{*}-x_{t}\right\rangle \\
& \geq \frac{t}{1-t}\left\langle\gamma f\left(x_{t}\right)+(I-B) S x_{t}\right. \\
& \left.\quad-A x_{t}, y^{*}-x_{t}\right\rangle, \quad y^{*} \in F .
\end{aligned}
$$

However, since $A-S$ is monotone,

$$
\left\langle(A-S) y^{*}, y^{*}-x_{t}\right\rangle \geq\left\langle(A-S) x_{t}, y^{*}-x_{t}\right\rangle .
$$

Combining the last two relations yields

$$
\begin{aligned}
& \left\langle(A-S) y^{*}, y^{*}-x_{t}\right\rangle \\
& \geq \frac{t}{1-t}\left\langle\gamma f\left(x_{t}\right)+(I-B) S x_{t}\right. \\
& \left.\quad-A x_{t}, y^{*}-x_{t}\right\rangle, \quad y^{*} \in F .
\end{aligned}
$$

Letting $t=t_{n} \rightarrow 0+$ as $n \rightarrow \infty$ in (49), we get

$$
\left\langle(A-S) y^{*}, y^{*}-x^{\prime}\right\rangle \geq 0, \quad y^{*} \in F .
$$

The equivalent dual VI of (50) is

$$
\left\langle(A-S) x^{\prime}, y^{*}-x^{\prime}\right\rangle \geq 0, \quad y^{*} \in F .
$$

Namely, $x^{\prime}$ is a solution of VI(13); hence $x^{\prime} \in \Omega$.

We further prove that $x^{\prime}=x^{*}$, the unique solution of VI(12). As a matter of fact, we have by (45)

$$
\begin{aligned}
\left\|x_{t_{n}}-x^{\prime}\right\|^{2} \leq & \frac{1}{\tilde{\lambda}+\tilde{\gamma}-\gamma \rho} \\
& \times\left\langle\gamma f\left(x^{\prime}\right)+(I-B) S x^{\prime}-A x^{\prime}, x_{t_{n}}-x^{\prime}\right\rangle, \\
& \quad x^{\prime} \in \Omega .
\end{aligned}
$$

Therefore, the weak convergence to $x^{\prime}$ of $\left\{x_{t_{n}}\right\}$ right implies that $x_{t_{n}} \rightarrow x^{\prime}$ in norm. Now we can let $t=t_{n} \rightarrow 0$ in (45) to get

$$
\left\langle\gamma f\left(y^{*}\right)+(I-B) S y^{*}-A y^{*}, y^{*}-x^{\prime}\right\rangle \leq 0, \quad \forall y^{*} \in \Omega,
$$

which is equivalent to its dual VI

$$
\left\langle\gamma f\left(x^{\prime}\right)+(I-B) S x^{\prime}-A x^{\prime}, y^{*}-x^{\prime}\right\rangle \leq 0, \quad \forall y^{*} \in \Omega .
$$

It turns out that $x^{\prime} \in \Omega$ solves $\operatorname{VI}(12)$. By uniqueness, we have $x^{\prime}=x^{*}$. This is sufficient to guarantee that $x_{t} \rightarrow x^{*}$ in norm, as $t \rightarrow 0+$. This completes the proof. 
Corollary 6. For each $(s, t) \in(0, \kappa) \times(0,1)$, let $\left\{x_{s, t}\right\}$ be a double net defined by

$$
\begin{aligned}
x_{s, t}=P_{C}[ & s\left(t \gamma f\left(x_{s, t}\right)+(1-t B) S x_{s, t}\right) \\
& \left.+(1-s) \frac{1}{\lambda_{s}} \int_{0}^{\lambda_{s}} T(\nu) x_{s, t} d \nu\right],
\end{aligned}
$$

for all $(s, t) \in(0, \kappa) \times(0,1)$. Then, for each fixed $t \in(0,1)$, the net $\left\{x_{s, t}\right\}$ defined by (55) converges in norm, as $s \rightarrow 0+$, to a solution $x_{t} \in F$. Moreover, as $t \rightarrow 0+$, the net $\left\{x_{t}\right\}$ converges in norm to $x^{*}$ which solves the following variational inequality:

$$
\begin{array}{r}
x^{*} \in \Omega, \quad\left\langle(I-\gamma f) x^{*}-(I-B) S x^{*}, x-x^{*}\right\rangle \geq 0, \\
\forall x \in \Omega,
\end{array}
$$

where $\Omega$ is the set of the solutions of the following variational inequality:

$$
x^{*} \in F, \quad\left\langle(I-S) x^{*}, x-x^{*}\right\rangle \geq 0, \quad \forall x \in F .
$$

Corollary 7. For each $(s, t) \in(0, \kappa) \times(0,1)$, let $\left\{x_{s, t}\right\}$ be a double net defined by

$$
x_{s, t}=P_{C}\left[s\left((1-t) S x_{s, t}\right)+(1-s) \frac{1}{\lambda_{s}} \int_{0}^{\lambda_{s}} T(\nu) x_{s, t} d \nu\right] \text {, }
$$

for all $(s, t) \in(0, \kappa) \times(0,1)$. Then, for each fixed $t \in(0,1)$, the net $\left\{x_{s, t}\right\}$ defined by (58) converges in norm, as $s \rightarrow 0+$, to a solution $x_{t} \in F$. Moreover, as $\rightarrow 0+$, the net $\left\{x_{t}\right\}$ converges to the minimum norm solution $x^{*}$ of the following variational inequality:

$$
x^{*} \in F, \quad\left\langle(I-S) x^{*}, x-x^{*}\right\rangle \geq 0, \quad \forall x \in F .
$$

Proof. In (55), we take $f=0$ and $B=I$. Then (55) reduces to (58). Hence, the net $\left\{x_{t}\right\}$ defined by (58) converges in norm to $x^{*} \in \Omega$ which satisfies

$$
x^{*} \in \Omega, \quad\left\langle x^{*}, x-x^{*}\right\rangle \geq 0, \quad \forall x \in \Omega .
$$

This indicates that

$$
\left\|x^{*}\right\|^{2} \leq\left\langle x^{*}, x\right\rangle \leq\left\|x^{*}\right\|\|x\|, \quad \forall x \in \Omega .
$$

Therefore, $x^{*}$ is the minimum norm solution of the VI(59). This completes the proof.

\section{Acknowledgment}

The author was supported in part by NSFC 71161001-G0105, NNSF of China (10671157 and 61261044), NGY2012097 and Beifang University of Nationalities scientific research project.

\section{References}

[1] I. Yamada, "The hybrid steepest descent method for the variational inequality problem over the intersection of fixed point sets of nonexpansive mappings," in Inherently Parallel Algorithms for Feasibility and Optimization and Their Applications, D. Butnariu, Y. Censor, and S. Reich, Eds., pp. 473-504, Elsevier, Amsterdam, The Netherlands, 2001.
[2] L.-C. Ceng, Q. H. Ansari, and J.-C. Yao, "Relaxed extragradient iterative methods for variational inequalities," Applied Mathematics and Computation, vol. 218, no. 3, pp. 1112-1123, 2011.

[3] L.-C. Ceng, Q. H. Ansari, and J.-C. Yao, "Some iterative methods for finding fixed points and for solving constrained convex minimization problems," Nonlinear Analysis. Theory, Methods \& Applications A, vol. 74, no. 16, pp. 5286-5302, 2011.

[4] L.-C. Ceng, S.-M. Guu, and J.-C. Yao, "A general iterative method with strongly positive operators for general variational inequalities," Computers \& Mathematics with Applications, vol. 59, no. 4, pp. 1441-1452, 2010.

[5] S.-S. Chang, H. W. Joseph Lee, and C. K. Chan, "A new method for solving equilibrium problem fixed point problem and variational inequality problem with application to optimization," Nonlinear Analysis. Theory, Methods \& Applications A, vol. 70, no. 9, pp. 3307-3319, 2009.

[6] S. S. Chang, H. W. Joseph Lee, C. K. Chan, and J. A. Liu, "A new method for solving a system of generalized nonlinear variational inequalities in Banach spaces," Applied Mathematics and Computation, vol. 217, no. 16, pp. 6830-6837, 2011.

[7] Y. J. Cho, I. K. Argyros, and N. Petrot, "Approximation methods for common solutions of generalized equilibrium, systems of nonlinear variational inequalities and fixed point problems," Computers \& Mathematics with Applications, vol. 60, no. 8, pp. 2292-2301, 2010.

[8] Y. J. Cho, S. M. Kang, and X. Qin, "On systems of generalized nonlinear variational inequalities in Banach spaces," Applied Mathematics and Computation, vol. 206, no. 1, pp. 214-220, 2008.

[9] F. Cianciaruso, V. Colao, L. Muglia, and H.-K. Xu, "On an implicit hierarchical fixed point approach to variational inequalities," Bulletin of the Australian Mathematical Society, vol. 80, no. 1, pp. 117-124, 2009.

[10] V. Colao, G. L. Acedo, and G. Marino, "An implicit method for finding common solutions of variational inequalities and systems of equilibrium problems and fixed points of infinite family of nonexpansive mappings," Nonlinear Analysis. Theory, Methods \& Applications A, vol. 71, no. 7-8, pp. 2708-2715, 2009.

[11] R. Glowinski, Numerical Methods for Nonlinear Variational Problems, Springer, New York, NY, USA, 1984.

[12] P. Kumam, N. Petrot, and R. Wangkeeree, "Existence and iterative approximation of solutions of generalized mixed quasivariational-like inequality problem in Banach spaces," Applied Mathematics and Computation, vol. 217, no. 18, pp. 7496-7503, 2011.

[13] M. Aslam Noor, "Some developments in general variational inequalities," Applied Mathematics and Computation, vol. 152, no. 1, pp. 199-277, 2004.

[14] M. A. Noor, "An extragradient algorithm for solving general nonconvex variational inequalities," Applied Mathematics Letters, vol. 23, no. 8, pp. 917-921, 2010.

[15] H.-K. Xu, "Viscosity method for hierarchical fixed point approach to variational inequalities," Taiwanese Journal of Mathematics, vol. 14, no. 2, pp. 463-478, 2010.

[16] I. Yamada, N. Ogura, and N. Shirakawa, "A numerically robust hybrid steepest descent method for the convexly constrained generalized inverse problems," in Inverse Problems, Image Analysis, and Medical Imaging, vol. 313 of Contemporary Mathematics, pp. 269-305, American Mathematical Society, Providence, RI, USA, 2002. 
[17] Y. Yao, Y. J. Cho, and R. Chen, "An iterative algorithm for solving fixed point problems, variational inequality problems and mixed equilibrium problems," Nonlinear Analysis. Theory, Methods \& Applications A, vol. 71, no. 7-8, pp. 33633373, 2009.

[18] Y. Yao, R. Chen, and H.-K. Xu, "Schemes for finding minimumnorm solutions of variational inequalities," Nonlinear Analysis. Theory, Methods \& Applications A, vol. 72, no. 7-8, pp. 34473456, 2010.

[19] Y. Yao, Y. J. Cho, and Y.-C. Liou, "Iterative algorithms for hierarchical fixed points problems and variational inequalities," Mathematical and Computer Modelling, vol. 52, no. 9-10, pp. 1697-1705, 2010.

[20] Y. Yao and J.-C. Yao, "On modified iterative method for nonexpansive mappings and monotone mappings," Applied Mathematics and Computation, vol. 186, no. 2, pp. 1551-1558, 2007.

[21] H. Zegeye, E. U. Ofoedu, and N. Shahzad, "Convergence theorems for equilibrium problem, variational inequality problem and countably infinite relatively quasi-nonexpansive mappings," Applied Mathematics and Computation, vol. 216, no. 12, pp. 3439-3449, 2010.

[22] H. Zegeye and N. Shahzad, "A hybrid scheme for finite families of equilibrium, variational inequality and fixed point problems," Nonlinear Analysis. Theory, Methods \& Applications A, vol. 74, no. 1, pp. 263-272, 2011.

[23] L.-C. Zeng and J.-C. Yao, "Strong convergence theorem by an extragradient method for fixed point problems and variational inequality problems," Taiwanese Journal of Mathematics, vol. 10, no. 5, pp. 1293-1303, 2006.

[24] I. Yamada and N. Ogura, "Hybrid steepest descent method for variational inequality problem over the fixed point set of certain quasi-nonexpansive mappings," Numerical Functional Analysis and Optimization, vol. 25, no. 7-8, pp. 619-655, 2004.

[25] N. Buong, "Strong convergence theorem for nonexpansive semigroups in Hilbert space," Nonlinear Analysis. Theory, Methods \& Applications A, vol. 72, no. 12, pp. 4534-4540, 2010.

[26] R. Chen and H. He, "Viscosity approximation of common fixed points of nonexpansive semigroups in Banach space," Applied Mathematics Letters, vol. 20, no. 7, pp. 751-757, 2007.

[27] R. Chen and Y. Song, "Convergence to common fixed point of nonexpansive semigroups," Journal of Computational and Applied Mathematics, vol. 200, no. 2, pp. 566-575, 2007.

[28] F. Cianciaruso, G. Marino, and L. Muglia, "Iterative methods for equilibrium and fixed point problems for nonexpansive semigroups in Hilbert spaces," Journal of Optimization Theory and Applications, vol. 146, no. 2, pp. 491-509, 2010.

[29] A. T. M. Lau, "Semigroup of nonexpansive mappings on a Hilbert space," Journal of Mathematical Analysis and Applications, vol. 105, no. 2, pp. 514-522, 1985.

[30] A. T.-M. Lau and W. Takahashi, "Fixed point properties for semigroup of nonexpansive mappings on Fréchet spaces," Nonlinear Analysis. Theory, Methods \& Applications A, vol. 70, no. 11, pp. 3837-3841, 2009.

[31] A. T.-M. Lau and Y. Zhang, "Fixed point properties of semigroups of non-expansive mappings," Journal of Functional Analysis, vol. 254, no. 10, pp. 2534-2554, 2008.

[32] H. Zegeye and N. Shahzad, "Strong convergence theorems for a finite family of nonexpansive mappings and semigroups via the hybrid method," Nonlinear Analysis. Theory, Methods \& Applications A, vol. 72, no. 1, pp. 325-329, 2010.
[33] T. Shimizu and W. Takahashi, "Strong convergence to common fixed points of families of nonexpansive mappings," Journal of Mathematical Analysis and Applications, vol. 211, no. 1, pp. 7183, 1997.

[34] K. Goebel and W. A. Kirk, Topics in Metric Fixed Point Theory, vol. 28 of Cambridge Studies in Advanced Mathematics, Cambridge University Press, Cambridge, UK, 1990.

[35] X. Lu, H.-K. Xu, and X. Yin, "Hybrid methods for a class of monotone variational inequalities," Nonlinear Analysis. Theory, Methods \& Applications A, vol. 71, no. 3-4, pp. 1032-1041, 2009. 


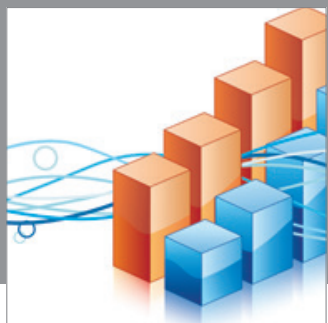

Advances in

Operations Research

mansans

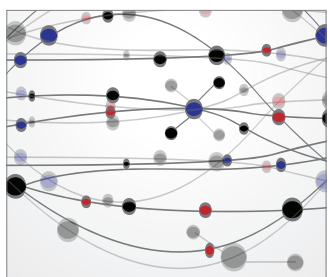

The Scientific World Journal
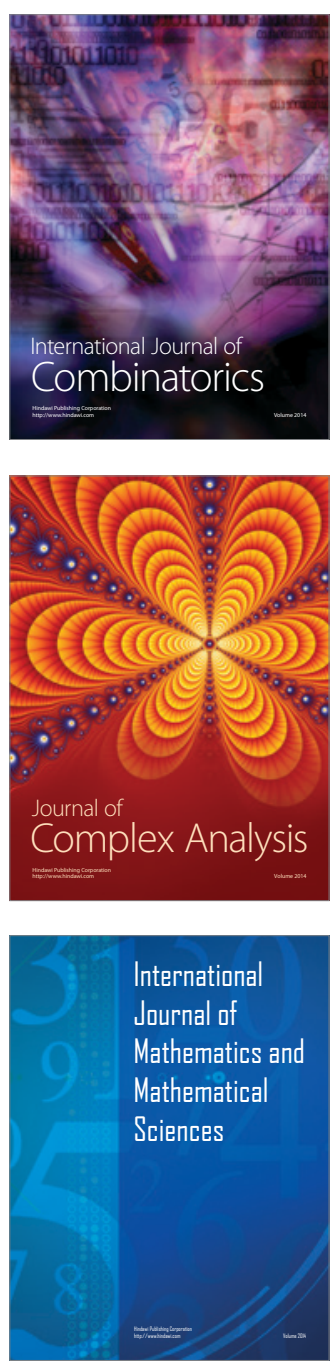
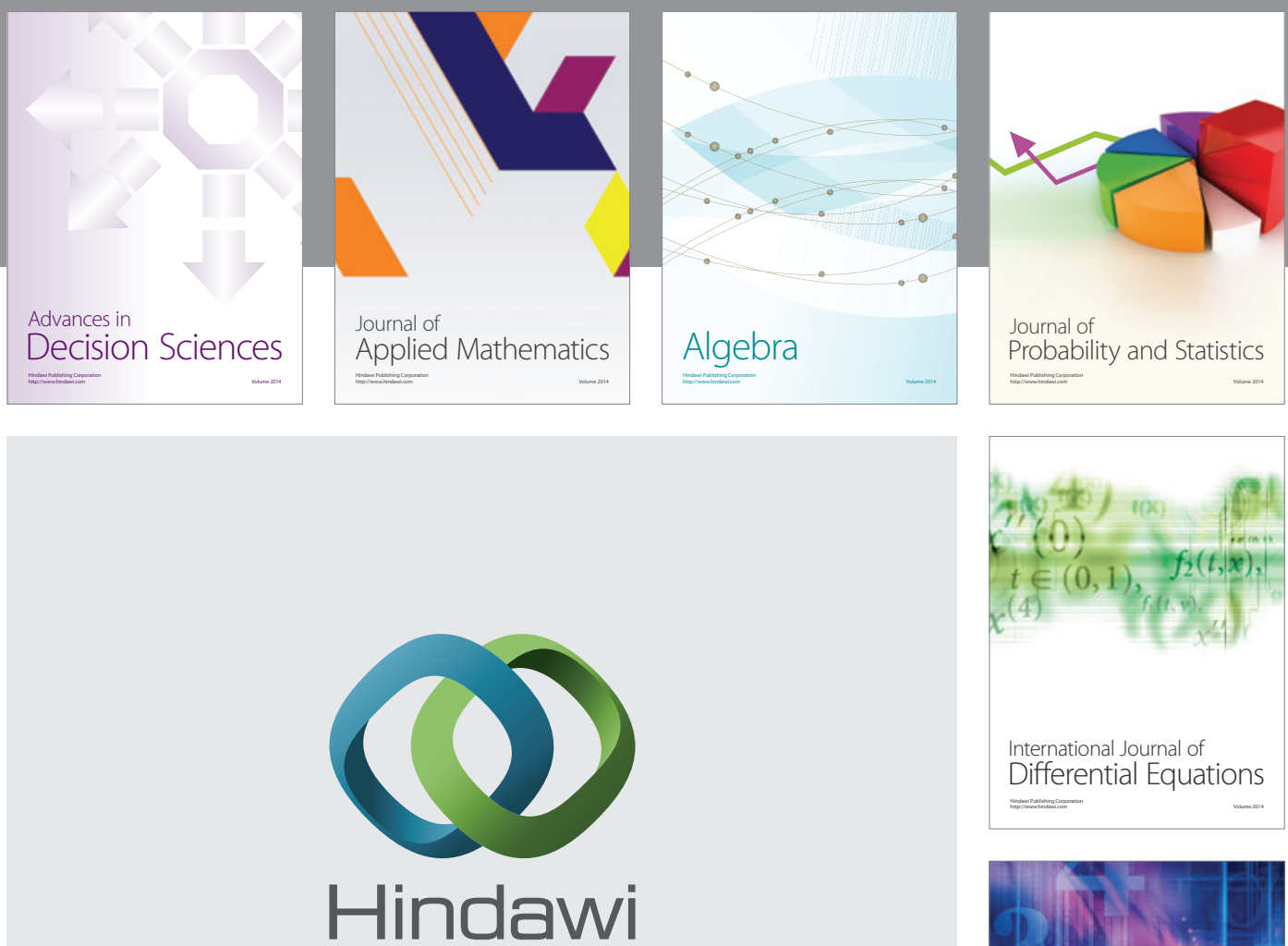

Submit your manuscripts at http://www.hindawi.com
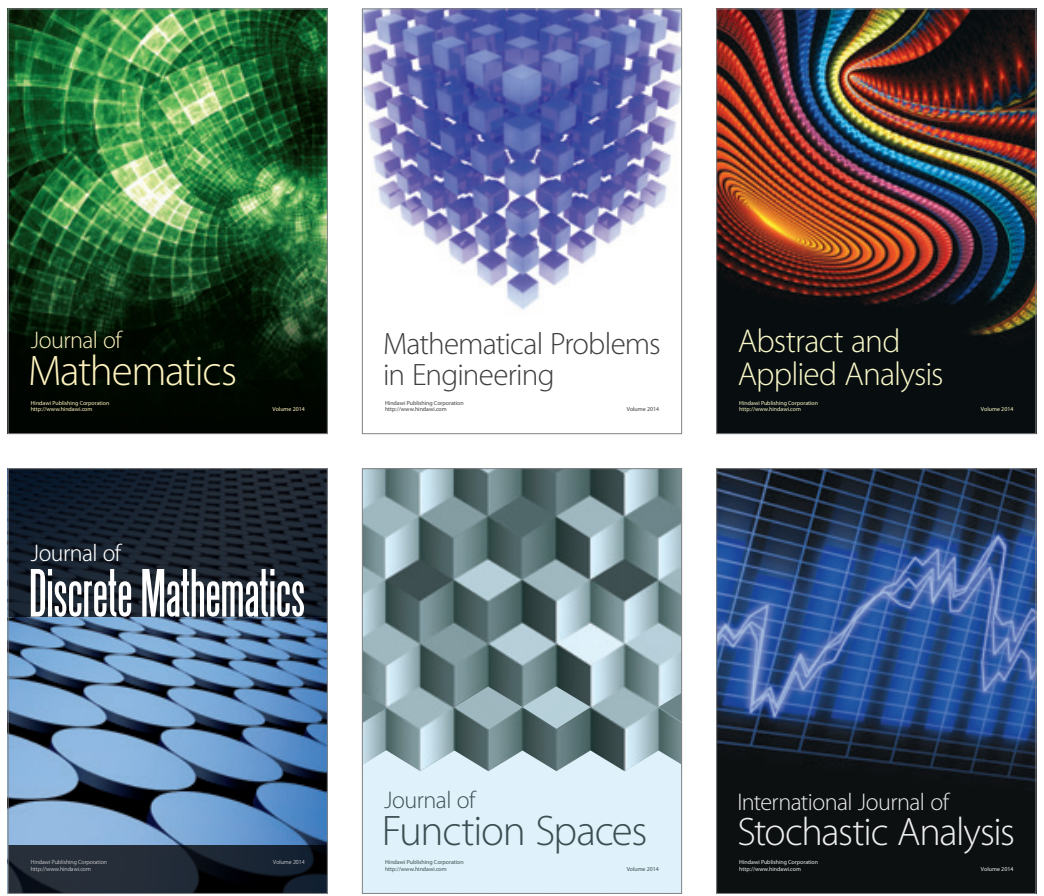

Journal of

Function Spaces

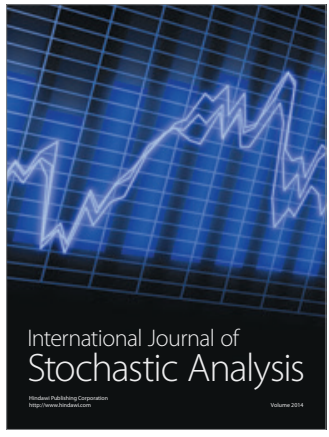

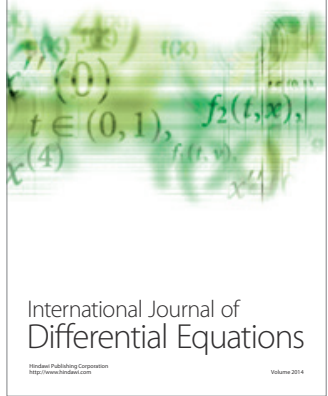
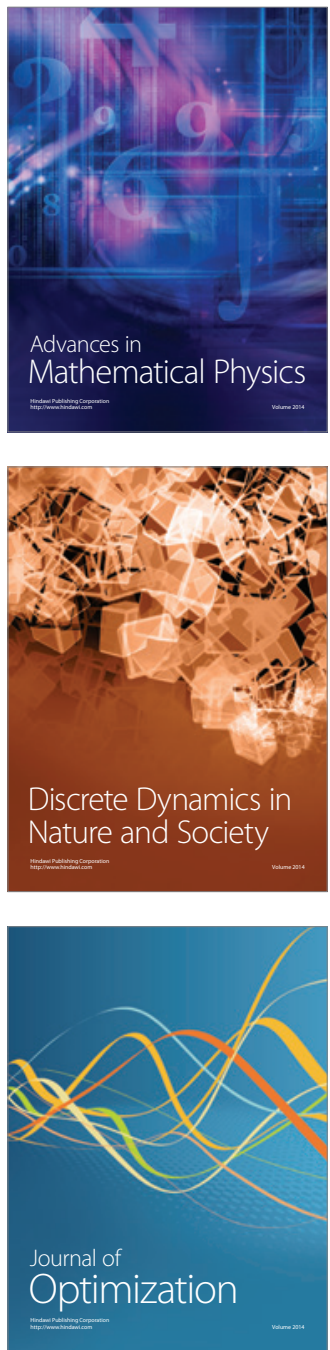